\title{
Implant and 3'S (Surface topography, Surface treatment, Sterilization)
}

\author{
Dr Mukti Chadda ${ }^{1}$, Dr Raghunath Patil ${ }^{2}$ \\ (Department of prosthodontics, V.K Institute of dental sciences, KLE university, Belgaum, India) ${ }^{1,2}$
}

\begin{abstract}
For centuries, clinicians have been attempting to replace missing teeth with suitable synthetic materials. Dental implants are fixtures that serve as replacements for the root of the missing natural tooth and becoming popular in the current day dental practice. Success or failure of the dental implant treatment is mainly based on the principles of creating and maintaining an interface between the implant and surrounding bone. This can be achieved by a phenomenon called osseointegration, which is the direct and stable anchorage of an implant due to the formation of bony tissue around the implant. A number of systemic and local factors influence the production of an osseointegrated interface and therefore the stability of the implant. However, surface characteristics of the implant materials in general and surface roughness in particular have received a great deal attention in the recent years to help achieve favourable interaction between the implant and biological tissues. Present article is a review of surface topography and treatments and its effect on the osseointegration of dental implant materials and also about sterilization of implants.
\end{abstract}

KEYWORDS; Implants, Osseointegration, Surface topography, Surface treatment, Sterilization.

\section{INTRODUCTION}

The field of biomaterials is of immense importance for the mankind as the very existence and longevity of some of the less fortunate human beings, who even at the time of birth are born with congenital defect and also for aged population who require biomedical implants to increase their life span. It is not new and as early as 4000 years back the Egyptians and Romans have used linen for sutures, gold and iron for dental applications. Nylon, Teflon, silicone, stainless steel and titanium were some of other materials which were put into use after World War II. ${ }^{1}$ Currently, the availability of better diagnostic tools and advancements in the knowledge on materials as well as on surgical procedures, implantology has assumed greater significance and bioimplants are commonly used in dentistry, orthopaedics, plastic and reconstructive surgery, ophthalmology, cardiovascular surgery, neurosurgery, immunology, histopathology, experimental surgery, and veterinary medicine.

Foremost requirement for choice of the biomaterial is its acceptability by human body. Implanted material should not cause any adverse effects like allergy, inflammation and toxicity either immediately after surgery or under post operative conditions. Secondly, biomaterials should possess sufficient mechanical strength to sustain the forces to which they are subjected so that they do not undergo fracture and more importantly, a bioimplant should have very high corrosion and wear resistance in highly corrosive body environment and varying loading conditions, apart from fatigue strength and fracture toughness. ${ }^{2}$

A biomaterial should remain intact for a longer period and should not fail until the death of the person. This requirement obviously demands a minimum service period of from 15 to 20 years in older patients and more than 20 years for younger patients. This can be achieved by taking into consideration three important factors that is surface topography, surface treatments and sterilization.

\section{SURFACE TOPOGRAPHY}

Implant surface character is one implant design factor affecting rate and extent of osseointegration .Process of osseointegration is now well described both histologically and at cellular level. Adhesion of a fibrin blood clot and population of implant surface by blood-derived cells and mesenchymal stem cells is orchestrated in a manner that results in osteoid formation and its subsequent mineralization. A seamless progression of changing cell populations and elaboration and modification of tissue/implant interface eventually results in bone forming in direct contact with the implant surface. Precisely how much of the implant surface directly contacts bone, how rapidly this bone accrual occurs mechanical nature of bone/implant connection is influenced by nature of implant surface itself.

Factors affecting osseointegration;

2.1) Implant Biocompatibility. 


\section{2) Design characteristics.}

2.3) Implant surface

\section{4) State of host bed}

\section{5) Surgical technique}

2.6) The loading conditions

\subsection{Implant biocompatibility;}

The modern concept of biocompatibility involves, not only compatibility of the material with tissue but its ability to perform a specific function. Response to specific individual materials could vary from one application site to another. Therefore, biocompatibility is defined only for a determined application. For example, the biomaterial used in intraocular lenses is not adequate for dental implants, although both show biological compatibility. In practice, there is no material that is biocompatible for all applications. Thus biocompatibility could not solely be dependent on the material properties but also had to be defined by the situation in which the material is used (William). ${ }^{3}$

The requirement for biocompatibility in a medical device intended for long term contact with the tissues of the human body is that the material does no harm to the tissues, and this presupposes chemical and biological inertness. However, in the case of dental implants, there is a need for osseointegration, which implies in a need for specific and direct interactions between biomaterials and tissue components. The main factors influencing the biocompatibility of biomaterials are chemical composition, mechanical properties, electrical charge and surface features. The biocompatibility of materials used in dental implants is evaluated by studying the reaction between the implant and the bone. The reaction between the implant and the soft tissue is also important. The region where the implant is in contact with the soft tissue is responsible establishing a kind of seal that isolates implant and the bone from the mouth environment. The analysis of the biocompatibility of biomaterials is accomplished by testing in vitro and complemented with tests in vivo. Tests in vitro involve cell culture, characterization of the chemical composition of surfaces, identification of the types of oxides and crystalline structures, determination of the thickness of the oxide layer, and quantification of resistance in mechanical loading conditions and dynamic resistance corrosion. The implant stability, surface roughness and surface wettability are also important. Implant tests in vivo are done in animals, especially mice, rabbits, dogs, sheep and pigs. The last testing stage involves analysis of human clinical trials. In tests in vivo, after implant removal from the animal, samples are analyzed by microscopy to determine tissue quality around implant.

In such tests certain types of tissue reaction have been repeatedly observed. Another important test is the interface implant-bone mechanical resistance after osseointegration of the implant. This measurement is indirectly determined by measuring the minimum torque required to remove the implant.

Tests in vivo are done to evaluate corrosion resistance and breakdown between toxicity, hijacking (non-vascular tissue and thick in contact with implant, the absence of pathological cells) and inertia (vascularised tissue and loose connective tissue). It is known that a relationship exists between material toxic reaction and a high corrosion rate. $\mathrm{Co}, \mathrm{Cu}, \mathrm{Ni}$ and $\mathrm{V}$ give rise to increased degradation in this order, and the degree of toxicity is increased in the same order. Metals that have corrosion passivation, i.e., high resistance to corrosion, do not trigger a negative response of tissues. This behavior is observed with $\mathrm{Pt}, \mathrm{Ta}, \mathrm{Nb}, \mathrm{Zr}$ and $\mathrm{Ti}$. It is possible to identify a correlation between biocompatibility, chemical stability and biological performance.

Corrosion resistance, degradation and corrosion products released are important parameters for materials selection . Morais et al. (Morais et al., 2009) evaluated the concentration of Ti, Al, and $\mathrm{V}$ in rabbit tissues (kidney, liver, and lung) after 1 week, 4 weeks, and 12 weeks of placement in the tibiae of Ti-6Al-4V orthodontic mini-implants. Various amounts of $\mathrm{Ti}, \mathrm{Al}$, and $\mathrm{V}$ were detectable, confirming diffusion of $\mathrm{Ti}, \mathrm{Al}$ and $\mathrm{V}$ from orthodontic mini-implants, with accumulation in remote organs (kidney, liver, and lung). Each metallic element had a different release behavior as a function of time. However, it is not enough to know which chemical elements are released; it is also important to analyze their influence on biocompatibility. Despite the tendency of metallic ion release with the Ti alloy, the measurable amounts of metals ( $\mathrm{Ti}, \mathrm{Al}$, and $\mathrm{V}$ ) released from Ti-6Al-4V orthodontic mini-implants in rabbits' tissues were significantly below the average dietary intake of these elements through food and drink and did not reach toxic concentrations. 
The analysis of biocompatibility becomes more complex when one considers that a low corrosion rate is not sufficient to ensure compatibility between implant and host tissue. Despite fact that the corrosion resistance of stainless steel is about 300 times greater than that of $\mathrm{Mg}$, many steel alloys release nickel, depending on the patient, can be highly toxic. A similar behavior problem is observed in $\mathrm{Cr}-\mathrm{Co}$ alloys (Williams, 2008). The amount of some chemicals that are released in body is a determinant factor of material biocompatibility. Evidences that there is correlation between the intrinsic toxicity of ions and their ability to bind macromolecules. It is necessary to study the ability of the products of corrosion of metals to react with host proteins to form a potentially toxic metal-protein, and to analyze stability of compounds formed during the release of corrosion products. A high corrosion resistance does not ensure biocompatibility; tissue reaction depends on the concentration and toxicity of corrosion products. Materials that ensure osseointegration are $\mathrm{cp}$ titanium, tantalum and niobium. Most companies employ cp titanium. Implants made of Ti-6\%Al-4\% $\mathrm{V}$ must be submitted to a surface treatment to exhibit osseointegration. ${ }^{4}$

\subsection{Design characteristics;}

There is not a consensus among researchers as to the best surface roughness and even the shape of the implants. The same manufacturer produces implants with different shapes and surfaces. It is common knowledge that the geometric shape of the implant should be chosen in such a way to provide the largest boneimplant contact area in order to improve osseointegration decrease the stress concentration close to cervical area. Commercial dental implants can be divided into groups according to shape, type of connection to the prosthetic component, surface treatment and roughness. ${ }^{5}$

\subsubsection{Shape: cylindrical, conical, hybrid;}

\subsubsection{Type of connection: external hexagon, internal hexagon, Morse taper, dodecagon;}

2.2.3Surface roughness: macro roughness, micro roughness (nanoroughness)

2.2.1 Shape ; In an attempt to improve the interaction between bone and implant, increase the surface area, the distribution of forces to the bone and achieve a better primary stability, various implants shapes were developed. Screw-shaped dental implants dominate the dentistry market. Screw shape provides a large contact area between implant and bone, increases primary stability, reduces shear stress in the bone-implant interface, reduces stress concentration in the cervical region and relieves stress concentration. Thread profile is characterized by the depth, pitch, flank angle, the top radius of curvature, and the straight part at the bottom of the thread. Rounded thread top relieves stress concentration and reduces stress on the bone. However, there is no recommendation or standardization for the screw of dental implants.

\subsubsection{TYPE OF CONNECTION;}

The original Brånemark protocol required several externally hexed implants to restore fully edentulous arches, linking them together via a metal bar with a fixed prosthesis. In this protocol, the external hex design was present to help screw the implant into place. In fact, for the fully edentulous, fixed prostheses abutments that were screwed down onto the implants, the hex was not engaged as an antirotation device. When the implants were later placed in single-tooth or partially edentulous cases, the hex had to be used to prevent rotation of the abutment and the single crown. External hex, which was only $0.7 \mathrm{~mm}$ in height, was not designed to withstand the forces directed on the crowns intraorally. Therefore, implant manufacturers had to compensate for this by changing the type of screw used (e.g., geometry, height, surface area), the precision of the fit over the hex, and the amount of torque used to secure the new screws. These changes have allowed externally hexed implants to be utilized with great confidence, although such efforts still require the clinician to radiographically verify that the abutments are fully seated. New interface designs are utilized on a variety of implants to improve the original external hex implant/ abutment interface. Goals of new designs are to improve connection stability throughout function and placement, and simplify the armamentarium necessary for clinician to complete the restoration.

\subsubsection{SURFACE ROUGHNESS;}

Sykaras et al has described three basic implant topographies;

A) Smooth B) Rough c) Porous

Smooth; surfaces with average $\mathrm{Sa}>1 \mathrm{um}$ are considered smooth

Rough: surfaces with $\mathrm{Sa}<$ are considered rough.

Primary aim of the surface texturing or treating the implant surface is to enhance cellular activity and improve bone apposition. Studies using endosseous dental implants in human clinical trials indicated that rough surfaces integrate better with the bone than those materials with relatively smooth surfaces . 


\section{SURFACE TREATMENTS}

A number of surface treatments are available to create controlled roughness on the surface of the implants. A) Addition B) subtraction procedures.

A plasma arc is a kind of addition process, which involves the deposition of bioactive hydroxyapatite material on the surface of the implants. Polishing, machining, and acid etching, on the other hand, are subtraction procedures.

Classification:

3.1 Mechanical,

3.2Chemical,

3.3Electrochemical,

3.4Electro polishing

3.5 Vacuum

3.6 Thermal

3.7Laser methods

3.1Mechanical methods involve treatment, shaping or removal of the material surface by means of physical forces. Mechanical treatments involve either removal of surface material by cutting or abrasive action, or the surface of the implant is deformed (and/or partially removed) by particle blasting. The most commonly employed mechanical techniques are machining, polishing, and blasting.

The properties of machined surfaces mainly depend on the work-piece speed, tool pressure and choice of lubricant. Machined implant surface is generally characterized by grooves and valleys more or less oriented along the machining direction and surface layers are plastically deformed.

Grinding creates relatively rough surface topographies. Grinding with an abrasive grade 60 leads to Ra values around $1 \mu \mathrm{m}$, and with the coarsest grade surface roughness's of up to 5-6 $\mu \mathrm{m}$ can be achieved.

Polishing of implant surface involves use of a fine abrasive material that is applied to a flexible wheel or a belt and then the implant is brought into direct contact with the abrasive surface. Polishing is always carried out in presence of lubricant. During the initial process coarse abrasive paper $(50-220$ grit $)$ is employed followed by a finer abrasive (about 600 grit) at a speed of $10-30 \mathrm{~m} / \mathrm{s}$. Polishing is generally carried out using $\mathrm{SiC}$, alumina or diamond to produce extremely smooth and mirror like surface with Ra values of $0.1 \mu \mathrm{m}$ or less. ${ }^{6}$

\subsection{Chemical treatments}

A variety of chemical treatments such as solvet cleaning, wet chemical etching, and passivation treatments have been employed for modifying the implant surfaces. Solvent cleaning is mainly aimed at cleaning the surface of the implant from oils, greases and fatty surface contaminants remaining after manufacturing process by using organic solvents (aliphatic hydrocarbons, alcohols, ketones or chlorinated hydrocarbons), surface active detergents and alkaline cleaning solutions. For affective cleaning process may be carried out at elevated temperatures with or without use of ultrasonication [2]. This process does not have any affect on surface of the implant. Selection of a solvent is based on the type of material to be cleaned and type of contamination to be removed from the material. Wet chemical etching dissolves native surface layer of the implant material including the oxide layer and parts of the underlying metal. Chemical etching is also used to improve the surface roughening as well as for producing an aesthetically favourable surface finish. Because titanium dioxide on the surface of the implant is a stable one, choice of etchants is limited to few acids and alkaline solutions. Acid etching or pickling is used for removing oxide layer to obtain clean and uniform surface finish. An aqueous mixture of 10-30 volume \% of nitric acid; (69 mass \%) and 1-3 volume\% of hydrofluoric acid $(60$ mass $\%)[2,23,30]$ is most commonly used etching solution. Relative proportion of nitric acid to hydrofluoric acid is critical as it minimizes formation of free hydrogen on reaction with titanium.

Formation of free hydrogen on surface of the implant embrittles implants. Mixture of $100 \mathrm{ml}$ hydrochloric acid (18 mass\%) and $100 \mathrm{ml}$ sulphuric acid (48 mass\%) can also be used as an alternative etchant to produce a significant surface roughness with micro pits of 1 - 10 ìm and large valleys of 20 - 30 um. Degree of pickling/etching is dependent on the acid concentration, temperature, and treatment time (typically in the range $1-60 \mathrm{~min}$ ). Surfaces which have been blasted prior to acid etching will generally show irregular surface topography. Sur-face roughnesses in the range from $0.1 \mu \mathrm{m}$ to several microns have been reported with this treatment. 
Alkaline etching is a simple technique to modify the titanium surfaces. Treatment of titanium in 4-5 M sodium hydroxide at $600 \mathrm{oC}$ for 24 hours has been shown to produce sodium titanate gel of 1 ìm thick, with an irregular topography with high degree of open porosity. Composition and structure of this layer can be further modified by proper heat treatment. Alternatively, boiling alkali solution $(0.2 \mathrm{M}$ sodium hydroxide, $1400 \mathrm{oC}$ for $5 \mathrm{~h}$ ) can be used to produce a high density of nanoscale pits on the titanium. When the alkali treatment is preceded by etching in hydrochloric acid/sulphuric acid, porosity of the final surface is found to increase.

Passivation treatments are used for obtaining a uniformly oxidized surface to improve corrosion resistance. It is often the last step in the surface preparation of implants. Immersion of titanium for a minimum of 30 minutes in 20-40 vol\% solution of nitric acid at room temperature is the most commonly employed method. After the passivation, surface of the implant should be neutralized, thoroughly rinsed and dried. ${ }^{7}$

\subsection{Electrochemical treatments}

Electro polishing and anodic oxidation, also known as anodizing, are the most commonly used methods for titanium surface modification. They are based on different chemical reactions occurring at an electrically energized surface placed in an electrolyte. The specimen to be treated is made the anode and by controlling the variables such as choice of electrolyte and other processing parameters such as electrode potential, temperature, current etc., to obtain different effects on the sample surface. ${ }^{8}$

\subsection{Vacuum treatments}

Vacuum treatment offers superior control on the processing conditions, especially with respect to cleanliness. Glow-discharge treatment, also known as cold plasma treatment, is based on the action of a lowpressure electrical discharge on the surface of the implant. Two different types of plasma treatments are available such as plasma deposition method and plasma surface modification. In plasma deposition, glow discharge is used to deposit the coating material from a separate solid target (sputter deposition) and/or by reactions in the gas phase (reactive sputtering or plasma polymerization). Plasma surface modification, on the other hand, is based on the exposure of sample surface to a glow di-charge in order to obtain a specific modification of surface properties. Surface modification of inorganic materials by cold plasma is achieved by bombardment of energetic ions, leading to removal of atoms and molecules from the surface (sputtering), and reactions between gas or plasma phase and surface atoms. Plasma treatment increases surface energy of the implant and there by improves the wetting characteristics as compared to conventional implant surfaces cleaned by using solvents or autoclaving. ${ }^{9}$

\subsection{Thermal treatments}

Commercially pure titanium was thermally annealed up to $1000^{\circ} \mathrm{C}$ to form oxide layer composed of anatase and rutile structures of $\mathrm{TiO} 2$ on the surface which is crack-free and uniformly rough. The average roughness of the oxidized surface observed when the titanium is annealed at $600 \mathrm{oC}$ and $650{ }^{\circ} \mathrm{C}$ for 48 hours was 0.90 and $1.30 \mathrm{im}$, respectively where as average roughness of untreated sample was $0.08 \mathrm{im}$. Thermal treatment at $600 \mathrm{oC}$ and $6500^{\circ} \mathrm{C}$ for 48 hours is considered appropriate for implanted materials. ${ }^{10}$

\subsection{Laser treatments}

Implant surface roughening using the previously discussed methods would cause surface contamination. Laser techniques have recently been developed as an alternative to these techniques. Laser enables implant surface treatment without direct contact and provides better control on the micro-topography of implant. Laser treatments are clean and easy method to perform. The average surface roughness of laser treated acid-etched implant was 2.28 ìm. Clinical studies have indicated more bone formation around the laser treated implants. This observation can be due to the formation of Tin on the surface that improves biocompatibility. ${ }^{11}$

\section{STERILIZATION;}

* Today in most cases manufacturers guarantees precleaned and presterilized implants, ready to be inserted. In case implants need to be resterilized conventional sterilization techniques are not satisfactory.

\subsection{Steam: Not be used as it results in contamination of surfaces with organic substances. 4.2Dry heat: Also leaves organic and inorganic surface residues. 4.3Radio frequency glow discharge technique (RFGDT) or plasma cleaning}

Proteintnaceous deposits and their action as films can be best eliminated by radio frequency glow discharge technique (RFGDT). Seems to be suitable for cleaning procedure. Implants are treated within a controlled noble, gas discharge at very low pressure. Gas ions bombard the surface and remove surface atoms and molecules, which are absorbed onto it or are constituents of it. Quality of surface treated depends on the gas 
purity. Baier et-al. showed that RFGDT is good for cleaning and at the same time, for granting a high energy state to implant, which is related to improved cell adhesion capabilities. Thinner, more stable oxide films and cleaner surfaces have been reported with RFGDT plus unproved wettability and tissue adhesion. The principal oxide at the surface is unchanged by the RFGDT process.

A decrease in bacterial contamination on HA-coated implant surfaces was reported after RFGDT, studies suggest that RFCDT may enhance calcium and/or phosphate affinity because of an increase in elemental zone at the surface resulting in the formation of amorphous calcium phosphate compounds.

\subsection{UV light sterilization}

Recently UV light sterilization is also being used. It cleans and increases the surface energy.

\subsection{Gamma radiation}

Method used to sterilize pre packaged dental implants. Radiation dose exceeding 2.5 megavolts is given

Components remain protected, clean and sterile until packaging is opened, within sterile field of surgical procedure. Some ceramics can be discolored and some polymers degraded by gamma radiation exposures. The limits are known for classes of biomaterials and all types of biomaterials can be adequately sterilized within the industry. Systems control, including pre-packaging and sterilization, has been an important part of the success of dental implantology.

\section{CONCLUSION;}

Various methods of surface modification or rough surface preparation in titanium and its alloys for implants were discussed with an emphasis on the methods based on the mechanical, thermal, chemical, electrochemical and laser methods. Although mechanical methods can be used to produce roughness on the titanium implant surfaces, properties of the surface oxide layer are more difficult to control. Chemical treatments of implant surface are mainly employed to improve the oxide layer thickness required for the passivation of the metal. Several alternative methods have been discussed which are used to produce surface films on titanium implants with varying morphology, thickness, microstructure, and chemical composition. In thermal treatments, surface roughness and amount of oxide layer formation are temperature and time dependent. Laser surface treatment, on the other hand, can used to produce desired surface roughness without any contamination of the implant surfaces.

\section{BIBLIOGRAPHY}

[1] Linkow implant dentistry today, vol 1; a multidisciplinary approach

[2] CARL E Misch; Contemporary Implant Dentistry, $2^{\text {nd }}$ edition

[3] Williams DF. (2008). On the mechanisms of biocompatibility. Biomaterials. Vol.29, pp. 2941,2953, ISSN 0142-9612.

[4] Morais LS, Serra GG, Palermo EFA, Andrade LR, Muller CA, Meyers MC, Elias CN. (2009). Systemic levels of metallic ions released from orthodontic mini-implants. American Journal Orthodontics Dentofacial Orthopedics. Vol.135, pp.:522-529. ISSN 08895406.

[5] Hanssen S, Norton M relationship between surface roughness and facial strength of bone .anchored implant. A Mathematical model .J Biomech 1999; 32; 829-836.

[6] Jan Eirik Ellingsen, Peter Thomsen, S. Petter Lyngstadaas, Advances in dental materials and tissue regeneration, Periodontology2000, $41,136-156$ (2006).

[7] Wen HB, Liu Q, de Wijin JR, de Groot K, Cui FZ, -Preparation of bioactive porous titanium surface by a new two-step chemicaltreatment- J Mater Sci: Mater Med, 9(3), 121 - 128 (1998)

[8] Kyo-Han KIM, Narayanan RAMASWAMY, Electrochemical surface modification of titanium in dentistry- Dent Mater J, 28(1),20-36 (2009).

[9] Yukari Shinonaga, Kenji Arita, Surface modification of stainless steel by plasma-based fluorine and silver dual ion implantation and deposition, Dental Materials Journal, 28(6), 735-742 (2009).

[10] Enori Gemelli, Alex Scariot, Nelson Heriberto Almeida Camargo, Thermal Characterization of Commercially Pure Titanium forDental Applications, Mater Res, 10 (3), 241-246 (2007).

[11] Marticorena M., G Corti, D Olmedo, M B Guglielmotti, S Duhalde, -Laser surface Modification of Ti implants to improveosseointegration, Journal of Physics: Conference Series 59, 662-665 (2007).

[12] 12)Doundoulakis J; Surface analysis of titanium after sterilization: Role in implant- tissue interface and bioadhesion. J Prosthet Dent; 1987 ; 54(4); 471-478. 